\title{
Interactions of ink colourants with chemically modified paper surfaces concerning inkjet print improvement
}

\author{
Sónia Sousa a , José António Gamelas ${ }^{\mathrm{b}, *}$, António de Oliveira Mendes ${ }^{\mathrm{a}}$, Paulo Torrão Fiadeiro ${ }^{\mathrm{a}, \mathrm{c}}$, \\ Ana Ramos ${ }^{\text {a }}$ \\ ${ }^{a}$ Research Unit of Textile and Paper Materials, University of Beira Interior, 6201-001 Covilhã, Portugal \\ ${ }^{\mathrm{b}}$ Chemical Engineering Department, University of Coimbra, Pólo II, 3030-790 Coimbra, Portugal \\ ${ }^{\mathrm{c}}$ Research Unit of Remote Sensing, University of Beira Interior, 6201-001 Covilhã, Portugal
}

\section{H I G H L I G H T S}

- The paper surface modification with surfactants and a cationic polymer was achieved.

- Paper modification with poly(diallyldimethylammonium) improved inkjet print quality.

- FTIR is of most helpful to access the functional groups of inkjet dyes and pigments.

- Sulphonate-containing dyes were better fixed on a polar surface with cationic groups.

- Low paper surface energy helped the inkjet pigments fixation at the paper surface.

\section{A R T I C L E I N F O}

\section{Article history:}

Received 12 June 2012

Received in revised form

4 January 2013

Accepted 18 February 2013

\section{Keywords:}

Interfaces

Surface properties

Adhesion

FTIR

ESCA

\begin{abstract}
A B S T R A C T
This study reports the modification of the paper surface by the application of different chemicals and its effect on the paper structural and surface properties and inkjet print quality parameters. 3- $(N, N-$ dimethylmyristylammonio)propanesulphonate and hexadecyltrimethylammonium bromide, as zwitterionic and cationic surfactants, respectively, and a cationic polymer, poly(diallyldimethylammonium) chloride (PD) were used for the surface treatments. It was found that, in general, the surface treatments have a negligible effect on the paper structural properties. However, a significant effect was found on the surface energy parameters, and surface chemical composition as accessed by XPS. The modified papers and the base paper were printed on two thermal inkjet printers: Lexmark X-8350 and HP Photosmart Pro B8850. The paper modified with PD exhibited the best print quality on both printers yielding high colour properties (gamut area increase of $12 \%$ and $11 \%$, respectively), good line and dot print quality. The print quality results were interpreted taking into account the nature (dyes or pigments) and molecular structure (determined by FTIR spectroscopy) of the inks colourants, paper surface properties, and the paper surface-ink interactions. Overall, the presence of cationic groups and a high polar component at the paper surface favour the fixation of sulphonate-containing dyes while a low paper surface energy and the evidence of aggregation/flocculating properties of the modifier (PD) helps the inks pigments immobilization.
\end{abstract}

(c) 2013 Elsevier B.V. All rights reserved.

\section{Introduction}

Inkjet has become a familiar word through its ubiquitous presence on the consumer desktop as a low cost, reliable, quick, and convenient method of printing digital files [1]. The inkjet print

\footnotetext{
* Corresponding author.

E-mail addresses: sonia.sousa@hotmail.com (S. Sousa), jafgas@eq.uc.pt (J.A. Gamelas), aomendes@ubi.pt (A. de Oliveira Mendes), fiadeiro@ubi.pt (P.T. Fiadeiro), ammr@ubi.pt (A. Ramos).
}

quality depends on the printer, inks physical-chemical properties and paper characteristics [2,3]. Important ink parameters are viscosity, surface tension, nature and molecular structure of colourants. Paper characteristics, such as surface topography and porosity, surface energy and the types of chemical groups present at the paper surface determine the ink setting and adhesion that contribute to the final print quality. For this reason, the "finishing" of the paper surface is extremely important concerning the print quality improvement. The surface finishing processes include chemical treatments such as surface sizing or coating and mechanical treatments such as calendering $[3,4]$. 
Most published studies regarding the paper surface treatments have been focused on the contribution of the coating to the inkjet print quality [5-14]. There are fewer studies published concerning the surface sizing and their impact on the inkjet print quality [15-22], and, for most of them, the interactions between the ink components and the paper surface have not been discussed with detail. It should be noted that surface sizing is of high importance for office papers, since these are generally uncoated but surface sized.

Surface sizing concerns the application of a formulation at the paper surface usually composed by starch and a hydrophobizing agent, with a typical dry solids content ranging from 2 to $15 \mathrm{wt} \%$ $[4,23]$. Other polymers such as modified starches, polyvinyl alcohol, sodium alginate, carboxymethyl cellulose, polyurethane, styreneacrylate and styrene-maleic anhydride copolymers, have also been used [3,15-17,20,24-26].

Recently, Saraiva et al. [19] studied the effect on the print quality of the paper surface modification with polyoxometalates. The application of these compounds afforded papers with high surface polarity, enabling the rapid diffusion of the ink vehicle and contributing to the dyes immobilization at the paper surface. The improvements obtained in the gamut area and optical density of cyan, magenta and yellow were attributed mainly to the presence of polyoxoanions and the corresponding counter-cations that provide a better fixation of the ink colourants at the paper surface.

It was reported that paper surface treatment with polymers based on glycol ethers together with starch and sizing agents improves mottling, strike-through and black density of the printing. In addition, with magnesium chloride, the same positive effect on the print quality parameters is obtained [18]. It was also mentioned the use of calcium chloride as fixing agent at the paper surface for the pigmented inks, improving CIE $L^{*} a^{*} b^{*}$ chroma and detail reproduction of the printed image [22]. According to Lundberg [22], pigmented inks often contain anionic dispersants to stabilize the ink and to avoid aggregation of particles. The use of metal salts on sizing formulations may change the ink stability by shielding the repulsive forces that stabilize the inks, thus making the pigments to aggregate faster at the paper surface.

Previous studies on the synthesis and use of new products in the surface sizing, namely, quaternary agents derived from fatty acids, led to positive results concerning the line and dot print quality comparatively to other synthetic agents. However, regarding colour reproduction, the results were not positive [16,21]. It was suggested that the low surface energy of the resultant papers, due to the aliphatic long chain molecules, allows the ink fixation on the paper surface, preventing the spreading and penetration, and thus yielding a good performance in terms of line and dot quality. However, the high acid component exhibited by those papers may explain the detrimental effect on the colour reproduction [21]. According to Zhmud [27], the paper surface acidity is one of the reasons for colour changes: in particular, it can change the ionization state of the colourant and the absorption spectra of the dye molecules.

The present work reports the influence of sizing formulations prepared with different commercial compounds on the paper properties (structural, surface and chemical) and their relation to the inkjet print quality parameters. The printers used to assess the print quality enable the evaluation of both dye and pigment-based inks, as well as the understanding of the physical-chemical interactions between the inks and the chemical compounds used for the paper surface modification.

\section{Experimental}

\subsection{Materials}

A base paper with $76.3 \mathrm{~g} \mathrm{~m}^{-2}$ basis weight, produced from bleached mixed softwood and hardwood kraft pulp and internally sized with alkenyl succinic anhydride (ASA) was used for the surface treatments. The following chemicals were selected for the paper surface modifications: native starch (supplied by the industry), 3-(N,N-dimethylmyristylammonio)propanesulphonate (Fluka, $\geq 98.0 \%$ purity), high molecular weight poly (diallyldimethylammonium) chloride solution (Aldrich), and hexadecyltrimethylammonium bromide (Sigma, $\geq 98.0 \%$ purity). The chemical structures of these compounds are shown in Fig. 1.

\subsection{Chemical modification of the paper surface}

All products for the paper surface chemical modification were used as received, except starch. The latter was submitted to a process of enzymatic conversion according to the procedure used in the paper mill. Formulations containing $12 \mathrm{wt} \%$ of solids content were prepared and applied, at room temperature, on one side of the base paper using a laboratory reverse roll coater (Mathis Type RRC$\mathrm{BW})$. The operation conditions of the coater were the following: distance between dip roll and applicator roll was $200 \mu \mathrm{m}$ except for the starch formulation $(125 \mu \mathrm{m})$; pressure between applicator and transport rolls was 3.5 bar, and roll speed was $20 \mathrm{~m} \mathrm{~min}^{-1}$. Sized papers were dried on an IR drier for $2 \mathrm{~min}$, after which time the temperature reached $100{ }^{\circ} \mathrm{C}$. The base paper and the modified papers were conditioned at $23^{\circ} \mathrm{C}$ and $50 \%$ relative humidity before characterization and printing.

The viscosity of the formulations was measured using a controlled stress rheometer (Haake R150). The measurements were performed at $23^{\circ} \mathrm{C}$ using a cone/plate geometry of $1^{\circ} / 20 \mathrm{~mm}$.

\subsection{Paper samples characterization}

Pick-up of the applied formulations was determined by weight difference of the paper before and after treatment according to the ISO 536:1995. Thickness (caliper), Bendtsen air permeability and Bekk smoothness of the paper sheets were determined according to ISO 534:2011, ISO 5636-3:1992 and ISO 5627:1995 standards, respectively.

Surface roughness parameters ( $R_{a}$ (arithmetic average height), $R_{q}$ (root mean square roughness), $R_{p}$ (maximum peak height) and $R_{v}$ (maximum valley depth)) were acquired using an Altisurf 500 optical profilometer coupled with the PaperMap 4.0 software. Each reported value corresponds to the mean of four 2D-profile analyses.

X-ray photoelectron spectra (XPS) were registered on a Kratos Axis Ultra DLD using a monochromatic $\mathrm{Al} \mathrm{K} \alpha$ radiation $(12 \mathrm{kV}$, $8 \mathrm{~mA}$ ). For each paper sample, a survey spectrum in the $0-1100 \mathrm{eV}$ binding energy range, using a $160 \mathrm{eV}$ pass energy, was recorded in two different locations. High-resolution spectra of the C1s and N1s regions were recorded at $20 \mathrm{eV}$ pass energy. The surface composition in atomic percentage was calculated for each analysed element $(\mathrm{C}, \mathrm{O}, \mathrm{N}, \mathrm{S}, \mathrm{Cl}, \mathrm{Br}, \mathrm{Ca}$ and $\mathrm{Si})$, by measuring the area of the corresponding peak.

Contact angles of the paper samples with deionised water, methylene iodide (Aldrich, 99\% purity) and ethylene glycol (Merck, $>99.5 \%$ purity) were measured with a DataPhysics OCAH 200 using the sessile drop method. In order to evaluate the apparent surface energy, the initial contact angle was measured after $c a .50 \mathrm{~ms}$ of the drop fall on the paper surface. This time may be considered short enough to avoid absorption and thus an equilibrium drop shape could be assumed. The apparent surface energy of the samples and the corresponding polar and dispersive components were calculated according to Owens, Wendt, Rabel and Kaelble approach [28]. The liquids surface tension values that were used for the calculations can be found in Ref. [29]. 

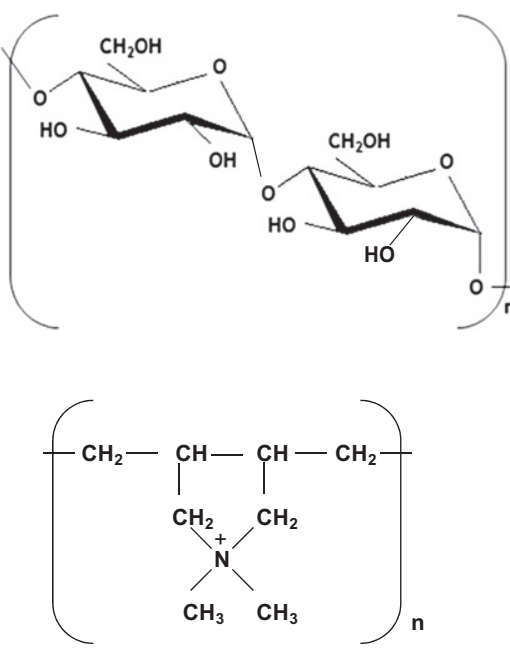

(a)

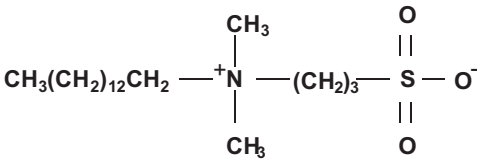

(b)<smiles>C[14C](C)(C)[N+](C)(C)Br</smiles>

(c)

\section{(d)}

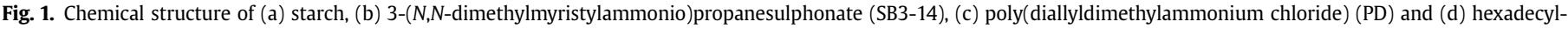
trimethylammonium bromide (CTAB).

\subsection{Inks characterization}

The inkjet inks used in the present study are aqueous based, that is, the solvent/dispersant is mainly water. Low amounts of alcohols may be present as well. The inks surface tension was evaluated by the pendant drop method using the OCAH 200 Dataphysics Instrument. Particle size was determined in inks samples diluted with ultrapure water using a Zetasizer Nano ZS from Malvern Instruments. The inks were also characterized by FTIR spectroscopy. For that, a drop of ink (about $10 \mu \mathrm{L}$ ) was placed on a $\mathrm{KBr}$ pellet, and the pellet was left drying in an exsiccator containing $\mathrm{P}_{2} \mathrm{O}_{5}$ before measuring. The infrared spectra were obtained using a Jasco FT/IR4200 spectrometer in the $400-4000 \mathrm{~cm}^{-1}$ range with a resolution of $4 \mathrm{~cm}^{-1}$ and a number of scans equal to 64 .

\subsection{Print evaluation}

A specific target was printed with an inkjet Lexmark X-8350 thermal printer that uses dye-based colours (35-XL) and pigmented black (34-XL), and with a HP Photosmart Pro B8850 printer using pigment-based inks (C9415A-C9417A and C9412A). The same print conditions were chosen for both: plain paper and best print quality. Afterwards, the inkjet print quality was evaluated by measuring colour, line and dot quality parameters.

Colour measurements were carried out with an AvaMouse handheld reflection spectrophotometer using the following conditions: D65 illuminant; observer, $2^{\circ}$; instrument geometry, $45^{\circ} / 0^{\circ}$ and Status I as spectral response. The gamut area was calculated as the area of $\left(a^{*}, b^{*}\right)$-hexagon, where $a^{*}$ and $b^{*}$ are the CIE $L^{*} a^{*} b^{*}$ coordinates obtained for each solid colour: cyan, magenta, yellow, red, green and blue. The optical density of the black, cyan, magenta and yellow as well as the paper optical density

Table 1

Pick-up values and effect of surface chemical modification on paper thickness and Bendtsen air permeability.

\begin{tabular}{llll}
\hline Paper & Pick up $\left(\mathrm{g} \mathrm{m}^{-2}\right)$ & Thickness $(\mu \mathrm{m})$ & $\begin{array}{l}\text { Air permeability } \\
\left(\mu \mathrm{ma}^{-1} \mathrm{~s}^{-1}\right)\end{array}$ \\
\hline Base & - & $106.4 \pm 1.58$ & $8.2 \pm 0.18$ \\
Starch & $3.5 \pm 0.22$ & $107.2 \pm 1.33$ & $2.2 \pm 0.50$ \\
SB3-14 & $1.8 \pm 0.25$ & $108.9 \pm 2.14$ & $8.0 \pm 0.28$ \\
PD & $4.9 \pm 0.30$ & $111.1 \pm 1.55$ & $7.5 \pm 0.16$ \\
CTAB & $2.0 \pm 0.27$ & $108.3 \pm 1.62$ & $7.5 \pm 0.23$ \\
\hline
\end{tabular}

were measured in order to determine the print density. This latter corresponds to the difference between the print optical density and the paper optical density.

The analysis of the line and dot quality parameters was performed with a QEA, personal image analysis system (PIAS-II), whose measurement principle is based on the ISO-13660 standard. Horizontal black line width, raggedness (a measure of the contour irregularity), and blur (characterizing the distinctness of the transition from line to background) were measured, whereas intercolour bleed (a measure of the spreading between two adjacent colours) was calculated from the black and yellow lines width. In addition, the black dot gain (increase of the dot size) and circularity (measuring the deviation of the dot shape relatively to a perfect circle) were also measured. The dot gain, expressed in percentage, corresponds to the difference between the measured and the theoretical dot diameter $(400 \mu \mathrm{m})$. The PIAS-II system was also used for acquiring the images of the red printing on the base paper and on the modified papers. The surface area covered by these images is $3.2 \mathrm{~mm} \times 2.4 \mathrm{~mm}$.

\section{Results and discussion}

\subsection{Paper samples characterization}

The effect of the surface chemical modifications on several paper structural properties is shown in Tables 1 and 2. Table 1 also shows the values of the pick-up obtained with the different formulations, ranging from 1.8 to $4.9 \mathrm{~g} \mathrm{~m}^{-2}$. This broad range should be mainly due to the different viscosity of the formulations employed, since their solids content was kept constant. In fact, the formulations containing PD and starch, which present the higher viscosity values (Table 3), afforded the higher pick-up. Accordingly

Table 2

Effect of surface chemical modification on paper roughness: Bekk smoothness and topographical parameters.

\begin{tabular}{llllrr}
\hline Paper & $\begin{array}{l}\text { Bekk } \\
\text { smoothness }(\mathrm{s})\end{array}$ & $R_{a}(\mu \mathrm{m})$ & \multicolumn{1}{l}{$R_{q}(\mu \mathrm{m})$} & \multicolumn{1}{l}{$R_{p}(\mu \mathrm{m})$} & \multicolumn{1}{l}{$R_{v}(\mu \mathrm{m})$} \\
\hline Base & $17.7 \pm 1.17$ & $2.5 \pm 0.02$ & $3.2 \pm 0.04$ & $8.3 \pm 0.37$ & $8.7 \pm 0.36$ \\
Starch & $14.3 \pm 1.33$ & $2.7 \pm 0.11$ & $3.5 \pm 0.13$ & $8.5 \pm 0.36$ & $11.1 \pm 0.62$ \\
SB3-14 & $15.5 \pm 1.06$ & $2.8 \pm 0.16$ & $3.5 \pm 0.21$ & $8.6 \pm 0.60$ & $11.0 \pm 0.87$ \\
PD & $12.3 \pm 1.12$ & $3.1 \pm 0.13$ & $3.8 \pm 0.15$ & $9.6 \pm 0.18$ & $11.1 \pm 0.40$ \\
CTAB & $15.2 \pm 1.13$ & $2.8 \pm 0.12$ & $3.6 \pm 0.11$ & $10.1 \pm 0.90$ & $10.4 \pm 0.54$ \\
\hline
\end{tabular}


Table 3

Viscosity of the formulations employed for the paper surface treatment (shear rate of $1000 \mathrm{~s}^{-1}$ ).

\begin{tabular}{ll}
\hline Formulation & Viscosity (Pa s) \\
\hline Starch & 0.030 \\
SB3-14 & 0.009 \\
PD & 0.038 \\
CTAB & 0.014 \\
\hline
\end{tabular}

the greater thickness was obtained for the paper sample with the highest pick-up, i.e., that one obtained with the PD formulation. Although the pick-up obtained with starch was significantly higher than that obtained with SB3-14 and CTAB formulations, the thickness of the paper sample modified with starch was in the same range of that obtained with SB3-14 and CTAB. This result may suggest that starch penetrates deeper into the paper structure blocking some pores, without changing appreciably the paper thickness. As a consequence of the effect of the pores filling by starch, a lower air permeability was obtained for the paper sample modified with starch in comparison to the base paper and all the other paper samples.

Concerning the macroroughness and microroughness given by the Bekk smoothness and topographical parameters, respectively, the results (Table 2 ) showed that the surface of the modified papers is slightly rougher than the base paper. This result could be due to the paper re-humidifying upon application of the formulation and subsequent drying. Changes in roughness, when the paper is exposed to water or simply to high humidity conditions, have been reported by Enomae and Lepoutre [30] and Mao et al. [31]. According to Enomae and Lepoutre, the structural alterations contribute largely for the surface changes and several hypotheses have been proposed to explain this effect. Among them, the fibres swelling causes shape reversion from ribbon to tube, the breakage of fibre-fibre bonds, and the relaxation of drying stress [30].

The $R_{q}$ parameter corresponds to the standard deviation of the distribution of profile heights about the mean surface, and for a Gaussian distribution, $R_{q} / R_{a}=(\pi / 2)^{0.5} \approx 1.25$ [31]. Since in the studied paper samples, $R_{q} / R_{a}$ ranged between 1.26 and 1.28 , it can be concluded that the papers' surface exhibit an approximate Gaussian distribution of profile heights. This means that the papers' surface is regular, not exhibiting a high proportion of extreme peaks and/or valleys. This is in agreement with the similar $R_{p}$ and $R_{v}$ values obtained for the different paper samples.

Fig. 2 shows the effects of the paper surface modifications on the elemental composition at the paper surface determined by XPS. As expected, carbon and oxygen were clearly the most abundant elements. In addition, all paper samples, including the base paper, showed a small amount of nitrogen on their surface. The presence of a low amount of nitrogen on the base paper surface may be attributed to the various chemicals used in the papermaking, such as starch, retention agents, optical brighteners, or even precipitated calcium carbonate (PCC) [32]. In fact, previously reported XPS studies on starch have shown trace amounts of nitrogen, possibly derived from protein [33,34]. The paper modified with PD exhibited the largest nitrogen amount, in agreement with the fact that PD presents the highest theoretical N/C atomic ratio (0.125) comparatively to the chemicals used in the other treatments, and also due to the high pick-up obtained with PD. Nevertheless, the N/C atomic ratio found for the different paper samples was very low (not higher than 0.03 ). This may be explained by the low amounts of formulations applied and to the partial penetration of the surface sizing compounds into depths superior to $10 \mathrm{~nm}$ (not assessed by XPS). Calcium was also detected at the surface of all paper samples (Fig. 2), including the base paper, due to the incorporation of PCC, as filler, during the papermaking process [35]. Elements such as sulphur, chlorine and bromine arose in the paper samples obtained by surface treatment with SB3-14, PD and CTAB, respectively, confirming the presence of the latter compounds at the paper surface. Finally, a small amount of silicon appeared in the paper sample treated with starch (Fig. 2), probably due to starch contamination [33].

In order to identify the chemical bonds involving carbon, the high-resolution XP spectra in the C1s region were deconvoluted into four peaks. $\mathrm{C} 1$ peak identifies carbon only linked to hydrogen or to carbon $(\mathrm{C}-\mathrm{C} ; \mathrm{C}-\mathrm{H} ; \mathrm{C}=\mathrm{C})$. $\mathrm{C} 2$ peak corresponds to ethers and alcohols $(\mathrm{C}-\mathrm{O})$; $\mathrm{C} 3$ peak refers to $\mathrm{O}-\mathrm{C}-\mathrm{O}$ or $\mathrm{C}=\mathrm{O}$ bonds. Finally, the $\mathrm{C} 4$ peak includes carboxyl and ester groups $(\mathrm{O}-\mathrm{C}=\mathrm{O})$. Table 4 lists the $\mathrm{O} / \mathrm{C}$ atomic ratios obtained from the values reported in Fig. 2 and the relative percentages of the different bonds with carbon atoms. Concerning the $\mathrm{O} / \mathrm{C}$ ratio, it was found that the paper modified with starch presents a slightly lower value than that of the base paper, while the other compounds provided paper samples with an even lower value. These results are in agreement with the molecular structure of the compounds used for the surface treatment (the theoretical $\mathrm{O} / \mathrm{C}$ atomic ratio is 0.83 for starch, 0.16 for SB3-14, while PD and CTAB compounds do not possess oxygen atoms in their molecular structure).

The relative amount of $\mathrm{C} 2$ was considerably higher than that of C1, C3 and C4 for all paper samples. This result is in agreement with Pykönen et al. [36] and is the outcome of the prevalence of $\mathrm{C}-\mathrm{O}$ bonds in cellulose/hemicelluloses. The surface treatments reduced the relative amount of C2, C3 and C4 and increased C1. In addition, the variations, relatively to the base paper, were more pronounced for the paper samples treated with SB3-14, PD and $\mathrm{CTAB}$. These results are a consequence of the lack of carbon to oxygen bonds in SB3-14, PD and CTAB compounds. Moreover, the long hydrocarbon chain in the SB3-14 and CTAB, and the ring structure included in the main chain of PD lead to higher percentages of $\mathrm{C} 1$ groups for the paper samples modified with these compounds.

Further confirmation of the presence of surfactants and PD at the paper surface could be obtained by high-resolution XPS in the N1s peaks region (Fig. 3). It was found that the nitrogen detected in SB3-14-, PD- and CTAB-modified papers is primarily associated to
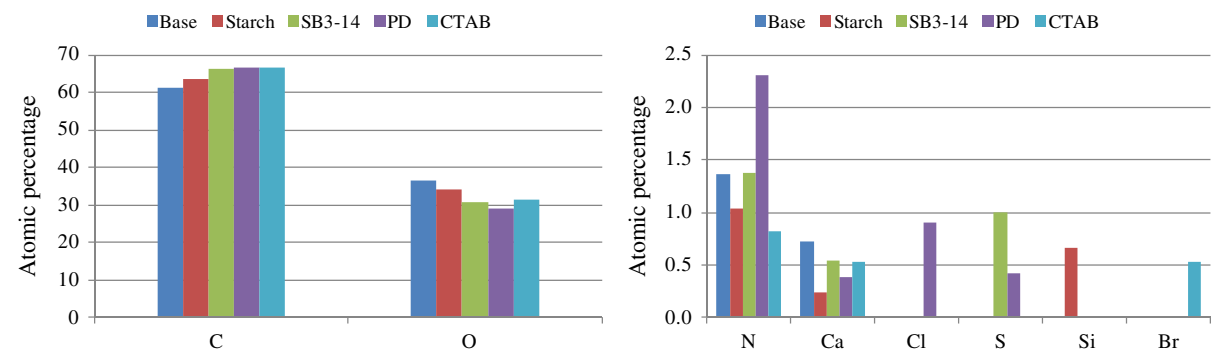

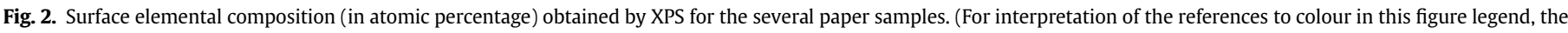
reader is referred to the web version of this article.) 
Table 4

Oxygen to carbon atomic ratio and relative percentage of C1-C4 groups for each paper sample.

\begin{tabular}{llllll}
\hline Paper & O/C & C1 (\%) & C2 (\%) & C3 (\%) & C4 (\%) \\
\hline Base & 0.59 & $20.9 \pm 0.24$ & $59.7 \pm 0.04$ & $15.9 \pm 0.06$ & $3.6 \pm 0.26$ \\
Starch & 0.54 & $28.7 \pm 2.03$ & $54.8 \pm 1.90$ & $13.9 \pm 0.59$ & $2.7 \pm 0.47$ \\
SB3-14 & 0.47 & $34.8 \pm 0.08$ & $51.5 \pm 1.06$ & $11.6 \pm 0.58$ & $2.1 \pm 0.40$ \\
PD & 0.44 & $31.9 \pm 0.45$ & $54.4 \pm 0.33$ & $11.8 \pm 0.28$ & $2.0 \pm 0.16$ \\
CTAB & 0.47 & $32.3 \pm 0.89$ & $52.9 \pm 1.27$ & $12.8 \pm 0.18$ & $2.1 \pm 0.20$ \\
\hline
\end{tabular}

$\mathrm{N}^{+} \mathrm{R}_{4}$ (quaternary ammonium) groups, while for the base paper and starch-modified paper, it corresponds only to $\mathrm{H}_{2} \mathrm{~N}-\mathrm{R}$ or $\mathrm{HN}-\mathrm{R}_{2}$ groups.

Fig. 4 illustrates the effects of the different compounds used for the paper surface treatment on the paper apparent surface energy and on its dispersive and polar components. The base paper is practically non-polar due to the internal sizing. The employed chemical modifications increased significantly the polar component of the surface energy and decreased the corresponding

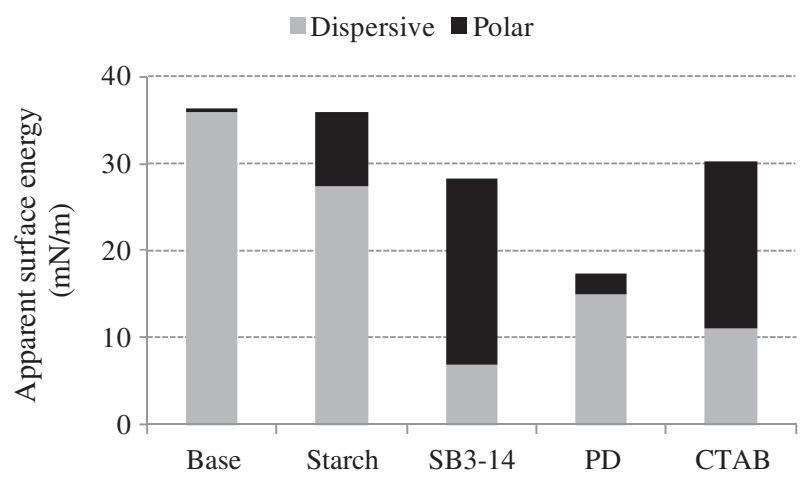

Fig. 4. Effect of the paper chemical modification on the apparent surface energy and on its dispersive and polar components.
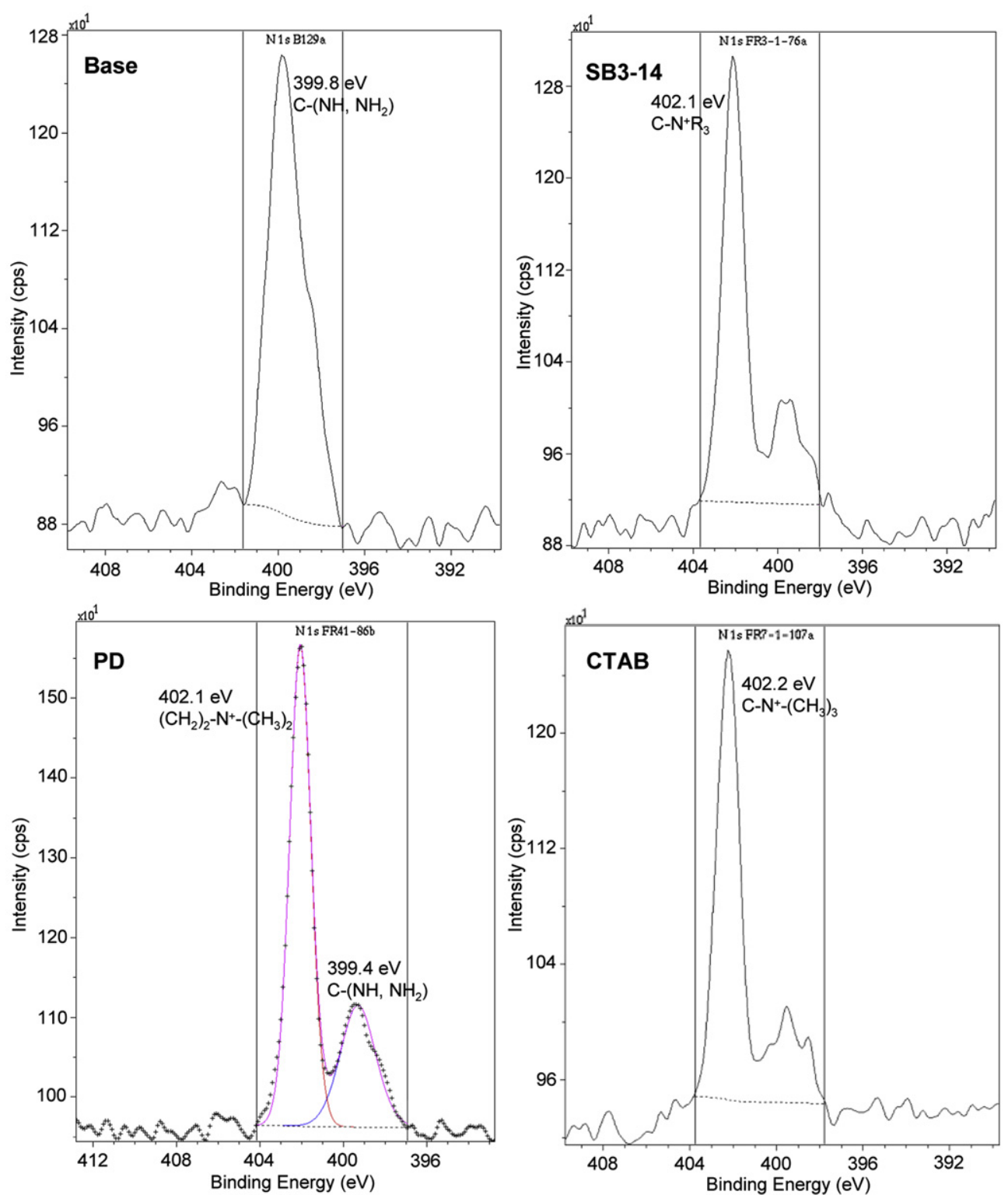

Fig. 3. XPS in the N1s region for several paper samples. 

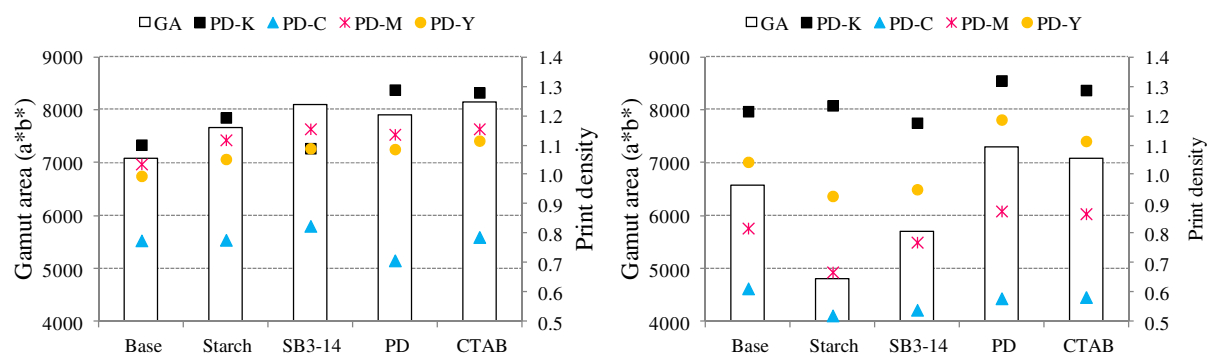

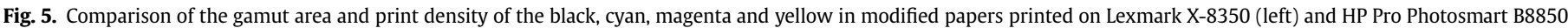
(right). (For interpretation of the references to colour in this figure legend, the reader is referred to the web version of this article.)

dispersive component. Concerning the starch, the high polar component of the surface energy is due to the high hydrophilicity of this polymer [34,37]. For the SB3-14 and CTAB-modified paper samples, the polar component was the largest contributor to the surface free energy. SB3-14 and CTAB are zwitterionic and cationic surfactants, respectively, constituted by a hydrophilic (polar) part and a hydrophobic tail (non-polar long hydrocarbon chain). Therefore, the obtained results suggest that the surfactant molecules link to the paper surface preferentially through hydrophobic groups, staying the hydrophilic groups directed towards the paper-air interface affording an extremely polar surface. For the PD-modified paper, besides the changes in the polar and dispersive component, a noticeable reduction in the total surface free energy occurred. PD is a highly charged linear polymer with a quaternary ammonium group in each chain unit $[38,39]$. Therefore, the reduction of the surface free energy, in comparison to the surfactant-modified paper samples, is consistent with the cationic polymer structure.

\subsection{Print evaluation}

Fig. 5 shows the gamut area and print density values obtained for the different paper samples printed on two printers. As shown, the surface modified papers printed on the Lexmark printer (left graphic) exhibited values of gamut area higher than that obtained with the base paper. On the other hand, the modified papers printed on the HP Photosmart printer (right graphic) provided

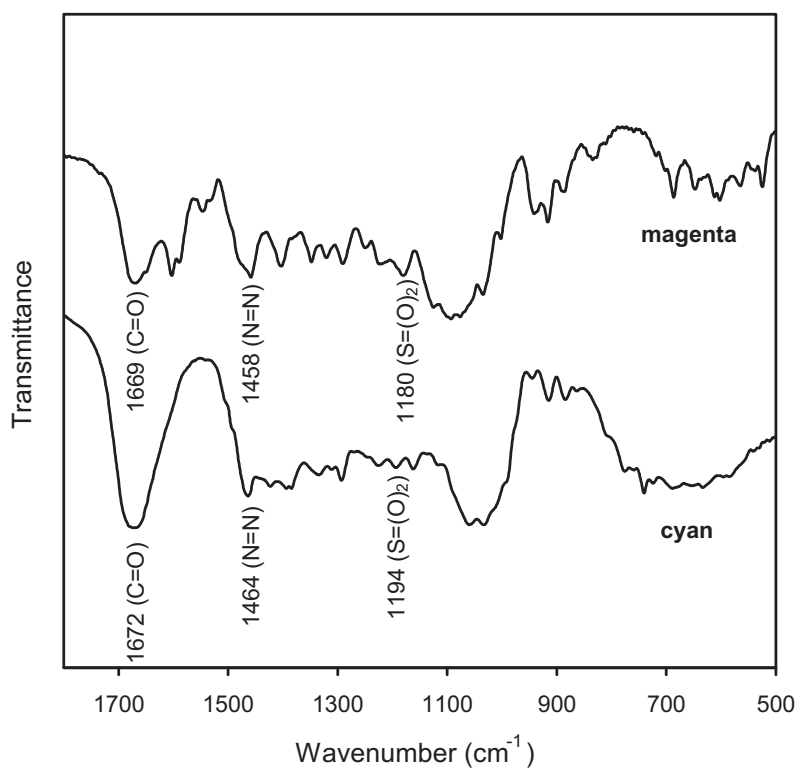

Fig. 6. FTIR spectra of cyan and magenta colours from Lexmark X-8350 (bands from $\mathrm{C}=\mathrm{O}$ and $\mathrm{N}=\mathrm{N}$ stretching and from $\mathrm{S}=(\mathrm{O})_{2}$ asymmetric stretching are highlighted). values of gamut area either upper or lower than that obtained with the base paper. Moreover, the HP Photosmart printer allowed a greater differentiation of the paper samples regarding colour quality. The best (higher) gamut area results for the Lexmark printer were obtained with the paper samples modified with SB314, PD and CTAB, while for the Photosmart printer, the PD and CTAB-modified papers clearly afforded the best gamut area values. In general, print density for cyan, magenta and yellow followed the same trend of the gamut area, i.e., papers with better colour reproduction also provided higher print density. However, black print density includes a few exceptions to the aforementioned trend, namely, the SB3-14-modified paper printed on the Lexmark printer and also the starch-modified paper printed on the HP Photosmart printer. It should be noted that the black print density trend along the several paper samples was similar for both printers.

The results obtained for the gamut area and print density may be explained taking into account the distinct colourants nature of the two printers considered (Lexmark uses dye-based inks, except for black, while HP Photosmart uses pigmented inks) and the different fixation mechanisms at the paper surface. To help understanding the paper surface-ink interactions, the inks were also characterized by FTIR spectroscopy, which can give an insight into the chemical nature of the ink chromophore compounds. FTIR spectroscopy (Fig. 6) showed that the Lexmark dyes (cyan, magenta and yellow) are azo compounds with an aromatic backbone containing sulphonate ions and carbonyl functional groups $[40,41]$. The black

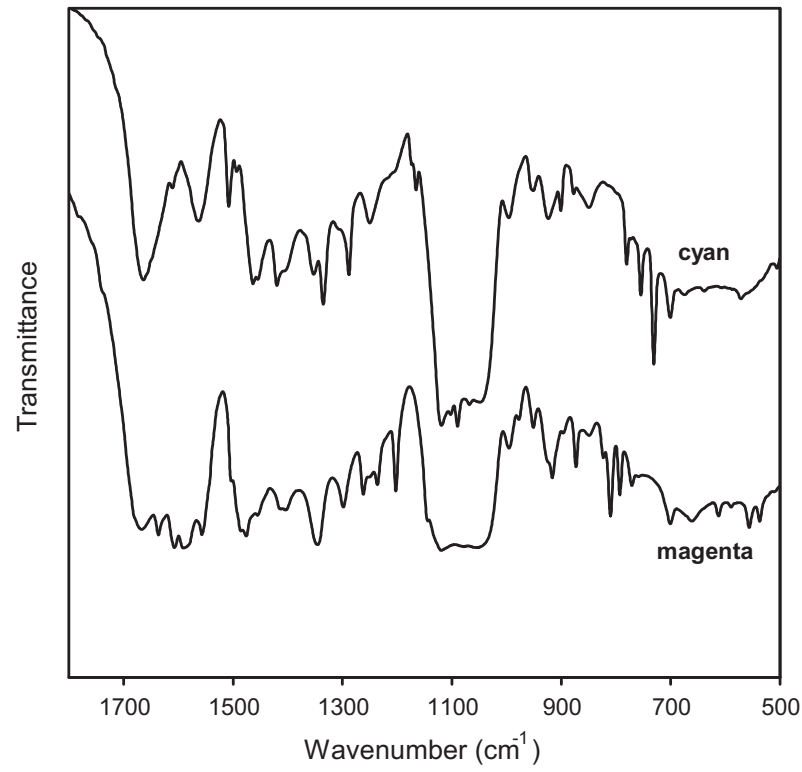

Fig. 7. FTIR spectra of magenta and cyan colours from HP Photosmart Pro B8850. The bands shown in the spectra fit perfectly with those of PR122 [44] (magenta) and PB15 [43] (cyan). 

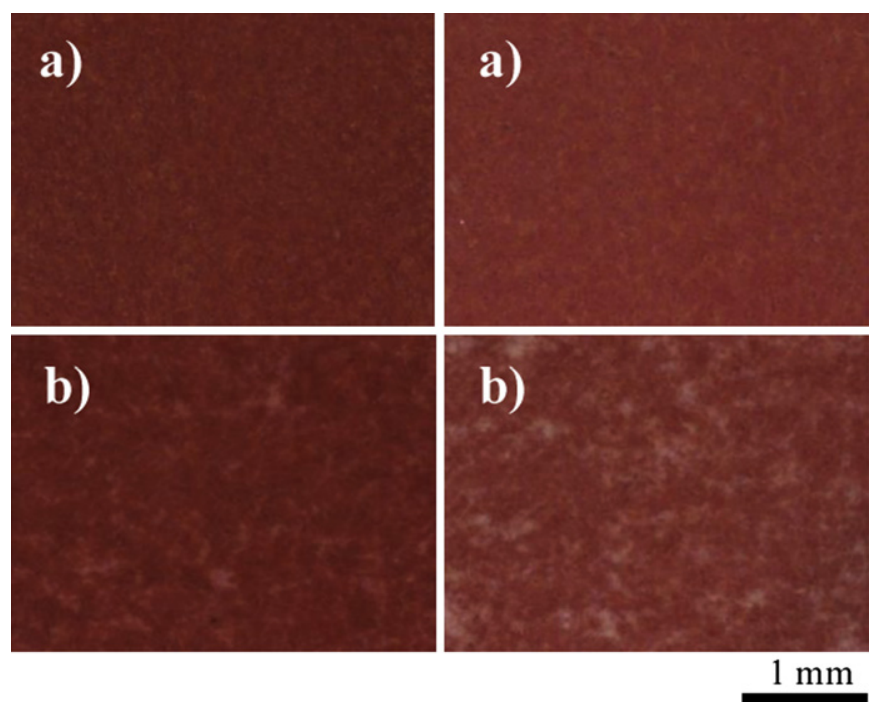

Fig. 8. Magnified images of red printing performed on Lexmark X-8350 (left) and HP Photosmart Pro B8850 (right) on: (a) base paper; (b) starch-modified paper. (For interpretation of the references to colour in this figure legend, the reader is referred to the web version of this article.)

pigment (with particle diameter of $c a .100 \mathrm{~nm}$ as determined using a Zetasizer) apparently does not contain sulphonate ions. Thus, the high electrostatic affinity between the anionic sulphonate groups present in the Lexmark (colour) inks composition and the positively charged groups of the quaternary amines may explain the better fixation of the colourants at the paper surface. As a result, higher values of the gamut area and optical density are obtained for the paper samples modified with SB3-14, PD and CTAB, in comparison to the base paper. Starch also provided an increase of gamut area and of the yellow and magenta optical density relatively to the base paper. The much lower surface porosity of the paper sample modified with starch (as shown above by the air permeability values) could suggest a lower penetration of the ink colourant into the paper structure [42], affording higher values of the optical density. However, when compared to the other treated paper samples, the gamut area and the optical density values have not improved. Thus, it is the interplay of paper surface physical parameters and paper surface chemistry that determines the colourant fixation vs. spreading or absorption. Besides coulombic forces other physical interactions, such as hydrogen bonds and van der Waals forces, in a lesser extent, may also contribute to the dyes fixation at the paper surface. In addition, the much higher polar component of the surface of the modified paper samples, in comparison to the base paper, should also help to separate the colourant from the vehicle (mainly water and, in minor amounts, alcohols).

Photosmart inks were also analysed by FTIR spectroscopy. From the FTIR data (Fig. 7) analysis, these pigmented inks (with particle size of $c a .80-130 \mathrm{~nm}$ ) contain copper phthalocyanine (PB15, blue), dimethylquinacridone (PR122, magenta) and an azo compound (yellow) $[41,43,44]$. The black ink contains carbon black. Apparently, based on the infrared data, the Photosmart pigments do not have sulphonate groups in their molecular structure (although for the yellow ink this could not be totally discarded). Thus, in the printing process, the presence of cationic groups at the paper surface should not be as effective as in Lexmark for achieving high gamut area or print density, as shown by the results obtained with the SB3-14-modified paper (Fig. 5). Taking into account both the surface energy and print quality results it can be suggested that, in general, a low apparent surface energy with a not very high polar component aids the colour pigment fixation by restricting the ink vehicle (controlling the spreading and absorption) at the paper surface. In the case of the PD-modified paper sample, the noticeable gamut area and print density results obtained with the HP Photosmart printer could be largely due to the destabilization effect of PD on the charged pigment colloidal particles of the aqueous ink formulation (it should be reminded that PD is a known flocculating agent used for instance in the wastewater treatment [45]), promoting their aggregation mechanism and thus also contributing to better fixing the pigment on the paper surface.

Surprisingly, the results obtained with starch (except for the black print density) were much poorer than those obtained with the base paper. Moreover, the paper sample modified with starch showed a distinct behaviour in the solid printed areas exhibiting non-uniform ink coverage, as depicted in Fig. 8 for the red printing. This effect, also shown for the Lexmark printing, although to a lesser degree, was very pronounced for the HP Photosmart printer, having a detrimental outcome in colour. This result could probably be due to the hydrophilicity and hypothetical penetration of starch into the paper structure, leading to the ink absorption in the regions where starch penetrates. Since the HP Photosmart inks have lower surface tension than the Lexmark inks (values of ca. $24 \mathrm{mN} \mathrm{m}^{-1}$ and $32 \mathrm{mN} \mathrm{m}^{-1}$, respectively, were found for colour inks) the ink absorption in the HP Photosmart printing should be higher resulting in a more evident non-uniformity. In contrast with the colour inks, the black print density did not worsen for the paper modified with starch in comparison to the base paper, which is in agreement with other studies recently published on the carbon black pigment penetration on paper samples surface sized with starch [46].

Fig. 9 shows the effect of the paper surface chemical modification on the black line print quality parameters namely width, blur, raggedness and intercolour bleed, and also on the black dot parameters such as gain and circularity. Comparing both diagrams, it is evident that the sequence in terms of line and dot quality was similar for the two printers. The paper modified with starch exhibited an inferior print quality followed by the SB3-14-modified paper. In opposition, the PD-modified paper produced the best print quality. From these results, it seems that, similarly to the colour pigments fixation, a lower apparent surface energy with a not very high polar component appears to be particularly
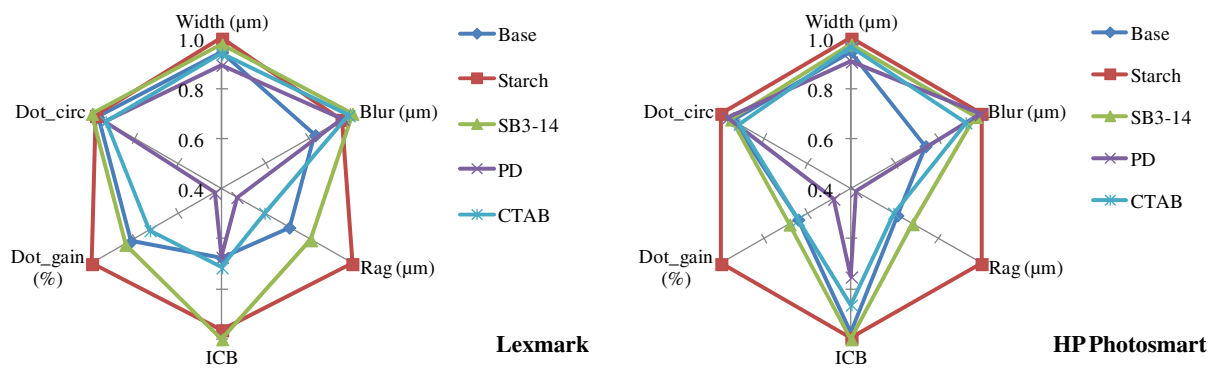

Fig. 9. Comprehensive effect on black line and black dot print quality parameters. Performance worsens from the chart centre. 
favourable for the black ink restraint on the paper surface, preventing it from spreading and, consequently, improving the line and dot print quality. This is especially true for parameters such as the line width, raggedness, intercolour bleed, and dot gain. The flocculating properties of PD must as well have contributed for the print quality improvement of the PD-modified paper. The dot circularity was not particularly affected by the chemical modifications, while it is clear that the line blur worsened. It may be proposed that the slight increase of roughness provided by the applied formulations contributes to the blur increase, i.e. worsens the line sharpness. It is interesting to note that the papers (PD and CTAB) with better performance on the line and dot parameters presented simultaneously a superior black print density. This confirms the importance of controlling spreading, in order to form a thick layer of ink on the paper surface, which contributes to a higher print density.

\section{Conclusions}

The effect of different surface chemical modifications on the paper structural and surface properties and on the inkjet print quality was evaluated. The evaluation of the performance of the new paper samples was made using two inkjet printers which allowed to access the behaviour of several dye- and pigment-based inks. The results showed that the paper surface modifications affected mainly its chemical properties, while the structural properties and surface topography undergone only slight variations. It was verified that the different surface chemical characteristics of the paper samples have a great impact on colour, black line and black dot print quality parameters. The paper samples with cationic charge and high polarity, when printed on Lexmark printer that uses dye-based colour inks (containing sulphonate anionic groups) resulted in high gamut area and high colour print density. To obtain high quality on colour, it is essential to fix the colourant on the paper surface. The immobilization of dye-based inks occurred mainly by ionic interactions, but hydrogen bonds and van der Waals forces could also be involved. On the other hand, other fixation mechanisms could operate for the pigment-based inks from the HP Photosmart printer (and for Lexmark black ink), such as (a) the pigment fixation by controlling the vehicle spreading (and absorption) due to a low surface energy with a not very high polar component of the paper sample or (b) the destabilization/aggregation of the pigment colloidal particles on the paper surface. Of all the produced paper samples, the one obtained by modification with poly(diallyldimethylammonium) (PD) exhibited the best print quality in both printers yielding high colour reproduction (gamut area increase of $12 \%$ and $11 \%$ in comparison to the base paper), high print density, limited line width with regular contours and lower intercolour bleed.

\section{Acknowledgements}

This work was carried out under the project PADIS (FCOMP-010202-FEDER-05348), which was co-financed by the European Union through FEDER (Fundo Europeu de Desenvolvimento Regional) in the extent of QREN (Quadro de Referência Estratégico Nacional 2007-2013) through COMPETE (Programa Operacional Factores de Competitividade).

The authors wish to acknowledge RAIZ - Institute of Forest and Paper Research for providing starch and base paper. They also thank the access to laboratory reverse roll coater and paper testing facilities.

The authors would also like to acknowledge LCPME - Laboratory of Physical Chemistry and Microbiology for the Environment, CNRS - National Centre of Scientific Research, Nancy Université, for the XPS analyses.

\section{References}

[1] A. Hudd, in: S. Magdassi (Ed.), The Chemistry of Inkjet Inks, World Scientific Publishing Co. Pte. Ltd., 2009, pp. 3-18.

[2] R.M. Xu, P.D. Fleming, A. Pekarovicova, V. Bliznyuk, J. Imaging Sci. Technol. 49 (6) (2005) 660-666.

[3] P. Fardim, Tappi J. 1 (7) (2002) 1-13.

[4] F. Tiberg, J. Daicic, J. Fröberg, in: K. Holmberg (Ed.), Handbook of Applied Surface and Colloid Chemistry, vol. 1, John Wiley \& Sons Ltd, 2001, pp. 123-173.

[5] E. Svanholm, Printability and Ink-coating Interactions in Inkjet Printing, PhD thesis, Karlstad University Studies, Karlstad, Sweden, 2007.

[6] H.-K. Lee, M.K. Joyce, P.D. Fleming, J.E. Cawthorne, Tappi J. 4 (2) (2005) 11-16.

[7] H.-K. Lee, M.K. Joyce, P.D. Fleming, J. Imaging Sci. Technol. 49 (1) (2005) 54-60.

[8] P.B. Malla, S. Devisett, Novel kaolin pigment for high solids ink jet coating, in: Proceedings of the PITA Coating Conference, Bradford, UK, 2005.

[9] J.Z. Shi, T.P. Schuman, J.O. Stoffer, J. Coat. Technol. Res. 1 (3) (2004) 225-234

[10] R. Sangl, J. Weigl, On the interaction between substrate and printing ink for ink-jet printing, in: Proceedings of the Tappi Printing and Graphic Arts Conference, Savannah, USA, 2000

[11] A. Hladnik, T. Muck, Dyes Pigment. 54 (2002) 253-263.

[12] J. Sreekumar, M. Sain, R. Farnood, W. Dougherty, Nord. Pulp Pap. Res. J. 22 (3) (2007) 307-313.

[13] T.T. Lamminmäki, J.P. Kettle, P.A.C. Gane, Colloids Surf. A 380 (2011) 79-88.

[14] T.T. Lamminmäki, J.P. Kettle, P.J.T. Puukko, P.A.C. Gane, Colloids Surf. A 377 (2011) 304-311.

[15] G.L. Batten Jr., Tappi J. 78 (1) (1995) 142-146.

[16] J.P. Koskela, O.E.O. Hormi, Appita J. 56 (4) (2003) 296-300.

[17] I.M.T. Moutinho, P.J.T. Ferreira, M.L.L. Figueiredo, Ind. Eng. Chem. Res. 46 (2007) 6183-6188.

[18] D.F. Varnell, U.S. Pat. 2008/0163993 A1, 10 Jul. 2008

[19] M.S. Saraiva, J.A.F. Gamelas, A.P.M. de Sousa, B.M. Reis, J.L. Amaral, P.J. Ferreira, Materials 3 (2010) 201-215.

[20] S. Sousa, A.P.M. de Sousa, B.M. Reis, A. Ramos, Influence of surface sizing with different binder systems on inkjet print quality, in: 2010 Symposium of Research Unit of Textile and Paper Materials, University of Beira Interior, Covilhã, Portugal, 2010

[21] S. Sousa, A.P.M. de Sousa, J.P. Koskela, A. Vaz, A. Ramos, Appita J. 63 (4) (2010) 300-307.

[22] A. Lundberg, Ink-paper Interactions and Effect on Print Quality in Inkjet Printing, Thesis for the Degree of Licentiate, Mid Sweden University, Sundsvall, Sweden, 2011.

23] L. Heikkilä, A. Tommeras, T. Engels, E. Knudsen, in: J. Gullichesen, H. Paulapuro (Eds.), Papermaking Science and Technology, Book 11, Fapet Oy, Helsinki, Finland, 2000, pp. 289-303.

[24] H.L. Lee, J.Y. Shin, C.-H. Koh, H. Ryu, D.-J. Lee, C. Sohn, Tappi J. 1 (1) (2002) 34-40.

[25] C. Andersson, L. Järnström, Distribution of starch and hydrophobic sizing agents, in: Proceedings of the 5th International Paper and Coating Chemistry Symposium, Montreal, Canada, 2003.

[26] P.D. Garrett, K.I. Lee, Tappi J. 81 (4) (1998) 198-203.

[27] B. Zhmud, Dynamic aspects of ink-paper interaction in relation to inkjet printing, in: Proceedings of the Pira International Printing Conference, Paper 4, Brussels, Belgium, 2003.

28] D.K. Owens, R.C. Wendt, J. Appl. Polym. Sci. 13 (1969) 1741-1747.

[29] R.J. Good, C.J. van Oss, in: M.E. Schrader, G.I. Loeb (Eds.), Modern Approaches to Wettability: Theory and Applications, Plenum Press, New York, 1991, pp. 1-27.

[30] T. Enomae, P. Lepoutre, Mechanism and dynamics of the roughening of pape in contact with moisture, in: Proceedings of the 4th International Symposium - Moisture and Creep Effects on Paper, Board and Containers, EFPG, Grenoble, France, 1999.

[31] C.Q. Mao, M.T. Kortschot, R. Farnood, J.K. Spelt, Nord. Pulp Pap. Res. J. 18 (1) (2003) 10-17.

32] P. Fardim, B. Holmbom, Appl. Surf. Sci. 249 (2005) 393-407.

[33] A. Rindlav-Westling, P. Gatenholm, Biomacromolecules 4 (2003) 166-172.

[34] H. Angellier, S. Molina-Boisseau, M.N. Belgacem, A. Dufresne, Langmuir 21 (2005) 2425-2433.

[35] P.J. Ferreira, J.A. Gamelas, I.M. Moutinho, A.G. Ferreira, N. Gómez, C. Molleda M.M. Figueiredo, Ind. Eng. Chem. Res, 48 (2009) 3867-3872.

[36] M. Pykönen, H. Sundqvist, J. Järnström, O.-V. Kaukoniemi, M. Tuominen, J. Lahti, J. Peltonen, P. Fardim, M. Toivakka, Appl. Surf. Sci. 255 (2008) 3217-3229.

[37] H.W. Maurer, R.L. Kearney, Starch 50 (1998) 396-402.

[38] M. Holmberg, in: J. Gullichesen, H. Paulapuro (Eds.), Papermaking Science and Technology, Book 4, Fapet Oy, Helsinki, Finland, 1999, pp. 223-239.

[39] Y. Zheng, A. Wang, J. Chem. Eng. Data 55 (2010) 3494-3500.

[40] C.W.M. Yuen, S.K.A. Ku, P.S.R. Choi, C.W. Kan, S.Y. Tsang, Res. J. Text. Apparel 9 (2005) 26-38

[41] N.P.G. Roeges, A Guide to the Complete Interpretation of Infrared Spectra of Organic Structures, Wiley \& Sons, Chichester, England, 1994.

[42] H. Nilsson, A. Fogden, Appita J. 61 (2008) 120-127.

[43] R. Newman, J. Am. Inst. Conserv. 19 (1979) 42-62.

[44] T.J.S. Learner, Analysis of Modern Paints, Getty Publications, Los Angeles, USA, 2004.

[45] J.K. Kapoor, S.Jabin, H.S. Bhatia, N. Kapoor,J. Chem. \& Cheml. Sci. 1 (2011)267-275.

[46] T.G. Costa, J.A. Gamelas, I.M. Moutinho, M.M. Figueiredo, P.J. Ferreira, Appita J. 63 (5) (2010) 392-398. 